\title{
NLRP3 inflammasome activation mediates radiation-induced pyroptosis in bone marrow-derived macrophages
}

\author{
Yan-gang Liu ${ }^{1,4}$, Ji-kuai Chen ${ }^{1,4}$, Zi-teng Zhang ${ }^{1,4}$, Xiu-juan Ma ${ }^{1}$, Yong-chun Chen ${ }^{1}$, Xiu-ming Du ${ }^{1}$, Hong Liu ${ }^{2}$, Ying Zong ${ }^{\star, 1}$ and \\ Guo-cai Lu, ${ }^{*, 1,3}$
}

\begin{abstract}
A limit to the clinical benefit of radiotherapy is not an incapacity to eliminate tumor cells but rather a limit on its capacity to do so without destroying normal tissue and inducing inflammation. Recent evidence reveals that the inflammasome is essential for mediating radiation-induced cell and tissue damage. In this study, using primary cultured bone marrow-derived macrophages (BMDM) and a mouse radiation model, we explored the role of NLRP3 inflammasome activation and the secondary pyroptosis underlying radiation-induced immune cell death. We observed an increasing proportion of pyroptosis and elevating Caspase-1 activation in 10 and 20 Gy radiation groups. NIrp3 knock out significantly diminished the quantity of cleaved-Caspase-1 (p10) and IL- $1 \beta$ as well as the proportion of pyroptosis. Additionally, in vivo research shows that 9.5 Gy of radiation promotes Caspase-1 activation in marginal zone cells and induces death in mice, both of which can be significantly inhibited by knocking out NIrp3. Thus, based on these findings, we conclude that the NLRP3 inflammasome activation mediates radiation-induced pyroptosis in BMDMs. Targeting NLRP3 inflammasome and pyroptosis may serve as effective strategies to diminish injury caused by radiation. Cell Death and Disease (2017) 8, e2579; doi:10.1038/cddis.2016.460; published online 2 February 2017
\end{abstract}

Radiotherapy is used extensively with bone marrow transplants and for restricting the growth and spread of a variety of tumors such as prostate cancer, lung cancer, and renal-cell carcinoma, etc. ${ }^{1-3} \mathrm{~A}$ limit to the clinical benefit of radiotherapy is not an incapacity to eliminate neoplastic cells but rather a limit on its capacity to do so without destroying normal tissue and inducing inflammation. ${ }^{4}$ Although the principal pathogenesis of radiation for damaging normal tissue is through the depletion of rapidly dividing cells, such as hematopoietic cells, vascular endothelial cells and somatic stem cells, emerging concepts suggest that exposure to radiation can also result in the activation and damage of peripheral immune cells and proinflammatory cytokine release, which in turn may impair the recovery and repopulation of destroyed cells and even result in a systemic response syndrome. ${ }^{4-6}$

Macrophages are recruited as a first response to radiationinduced damage. ${ }^{7}$ Alterations in macrophages following radiation have been observed during both early and late phases of tissue injury. ${ }^{8}$ Radiation polarizes macrophages towards an M1 phenotype, which is known as the pro-inflammatory phenotype, and enhances the secretion of M1 cytokines such as TNF-a, IL-12 and IFN- $\gamma$, which participate in pro-inflammatory responses. ${ }^{9,10}$ Activated M1 macrophages then promote extracellular matrix destruction, cell apoptosis, chronic inflammation (fibrosis) and tissue injury. ${ }^{4}$ Additionally, Raj et al. found that $\mathrm{N}$-acetyl tryptophan glucoside (NATG) pretreatment overcomes the radiation-induced immune response by affecting macrophages and thus contributes to radioprotection. ${ }^{11}$ Thus, to explore the mechanism of radiation-induced immune cell, particularly macrophage, activation or damage, it is of paramount importance to find ways to limit the side effects of radiation and to maximize future therapeutic benefit. In the present study, the mechanism of immune system damage under radiation was studied using primary cultured bone marrow-derived macrophages (BMDMs).

Mainly described in macrophages and dendritic cells, pyroptosis is recognized as a model of cell death distinct from apoptosis and is thought of as a regulated form of necrosis. ${ }^{12,13}$ Unlike apoptosis, pyroptosis is initiated by the recognizing of NOD-like receptors (NLRs) to pathogenassociated molecular patterns (PAMPs) and dangerassociated molecular patterns (DAMPs), leading to the assembly of a large multiprotein complex termed inflammasome which recruits and cleaves the precursor of Caspase- 1 (pro-Caspase-1, p45) to its active form. ${ }^{14,15}$ Active Caspase-1 then causes the enzymolysis of the precursors for inflammatory cytokines IL-1 $\beta$ and IL-18 or induces pore formation on the plasma membrane, which results in cell swelling and the release of cytosolic contents such as lactate dehydrogenase (LDH) and pro-inflammatory cytokines. ${ }^{16,17}$ However, the role of pyroptosis in radiation-induced macrophage damage remains unclear.

\footnotetext{
${ }^{1}$ Department of Health Toxicology, College of Tropical Medicine and Public Health, Second Military Medical University, Shanghai 200433, China; ${ }^{2}$ Radiology Department X-ray Room, The Second People's Hospital, Neijiang, Sichuan 641100, China and ${ }^{3}$ CTI Biotechnology (Suzhou) Co., Ltd, Jiangsu 215300, China

${ }^{*}$ Corresponding author: G-c Lu, Department of Health Toxicology, College of Tropical Medicine and Public Health, Second Military Medical University, NO 800, Xiang-yin Road, Shanghai 200433, China or CTI Biotechnology (Suzhou) Co., Ltd, Jiangsu 215300, China. Tel: +86 51 236802386; E-mail: newdrug@smmu.edu.cn or Y Zong, Department of Health Toxicology, College of Tropical Medicine and Public Health, Second Military Medical University, NO 800, Xiang-yin Road, Shanghai 200433, China. Tel: +86 21 81871035; E-mail: standyup @ hotmail.com

${ }^{4}$ These authors contributed equally to this work.

Received 12.9.16; revised 24.11.16; accepted 05.12.16; Edited by Y Shi
} 
In recent years, NLRP3 inflammasome upregulation at the expression or activation level has been reported to play an important role in radiation-induced lung inflammation, oral mucositis and skin lesions. ${ }^{18-21}$ Activation of Caspase-1 has also been linked with radiation exposure in the immune cells from spleen or hippocampal neural stem cells. ${ }^{5,22}$ Furthermore, Stoecklein et al. demonstrated that inflammasome activation occurs in many immune cell types following radiation exposure. ${ }^{5}$ As Caspase- 1 was found to be activated by radiation in many studies, whether the components downstream of active Caspase-1, such as pyroptosis, play a role in radiation-induced immune cell death remains unknown. In this study, using primary cultured BMDMs and a mouse radiation model, we reported here for the first time that NLRP3 inflammasome-mediated pyroptosis is of great significance in radiation-induced BMDM death.

\section{Results}

Radiation induced pyroptosis in bone marrow-derived macrophages. Cultured BMDM were exposed to a ${ }^{60} \mathrm{Co}$ radiation source to attain the desired doses of 5,10 and 20 Gy. After $24 \mathrm{~h}$, a cell viability assay was performed to evaluate the survival rate of cells. As shown in Figure 1a, groups exposed to radiation (5, 10 and $20 \mathrm{~Gy}$ ) illustrate a dose-dependent cell loss and reaches low levels by $10 \mathrm{~Gy}$ (cell viability $55.27 \%$ ) and 20 Gy (cell viability $45 \%, P<0.001$ versus $0 \mathrm{~Gy}$ ).
Pyroptotic cell death was assessed by measuring the release of $\mathrm{LDH}$ and double-positive staining of activated Caspase-1 and propidium iodide (PI). ${ }^{13}$ We detected that radiation (10 and $20 \mathrm{~Gy}$ ) induced increased activity of LDH dose dependently in BMDM; 10 and 20 Gy radiation induced activity of LDH from 1.318 to 2.442 and 2.782 (OD $490 \mathrm{~nm}$ ), respectively ( $P<0.001$ versus 0 Gy, Figure $1 \mathrm{~b})$. To determine whether the death of BMDM induced by radiation is a result of pyroptosis, we exposed BMDM to radiation (5, 10, and $20 \mathrm{~Gy}$ ) and detected activated Caspase-1 and PI using flow cytometry after $24 \mathrm{~h}$. As shown in Figures $1 \mathrm{c}$ and d, the proportion of double-positive (activated Caspase-1 and PI) BMDM (Q3) significantly increased in 10 Gy (29.66\%) and 20 Gy $(46.27 \%)$ radiation $(P<0.001$ versus $0 \mathrm{~Gy})$, while the $5 \mathrm{~Gy}$ group (9.674\%) showed no significant difference in comparison with the 0 Gy group $(5.604 \%, P>0.05$ versus $0 \mathrm{~Gy})$.

Radiation promoted pro-inflammatory cytokine production in bone marrow-derived macrophages. ELISA analysis of the pro-inflammatory cytokines in supernatant revealed that both 10 and 20 Gy radiation significantly increased the production of IL-1 $\beta$, IL-18, TNF- $\alpha$, IFN- $\gamma$, IL-1 $a, \mathrm{IL}-12 \mathrm{p} 40$ and MCP-1 (Figure 2). Notably, 5 Gy radiation also significantly induced the release of $\mathrm{IL}-1 \beta, \mathrm{IL}-18$ and IFN- $\gamma(P<0.05$, Figures $2 \mathrm{a}, \mathrm{b}$ and $\mathrm{e})$, but not TNF- $a$, IL-1a, IL-12p40 or MCP-1 $(P>0.05)$. Nevertheless, radiation stimulation did not have a significant effect on the production of IL-6 ( $P>0.05$, Figure $2 \mathrm{~h})$. Based on a number of studies of inflammasome activation, we used IL-1 $\beta$ instead of both a

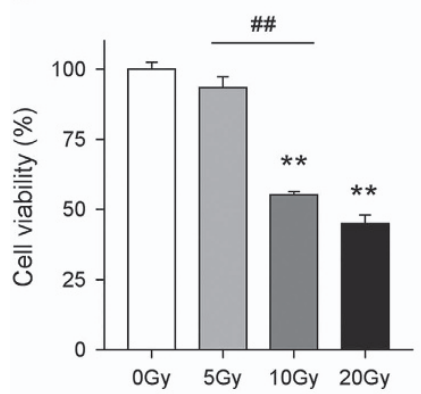

b

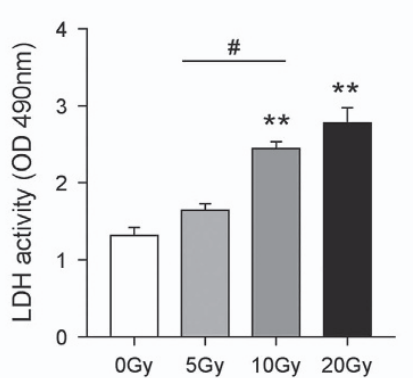

C

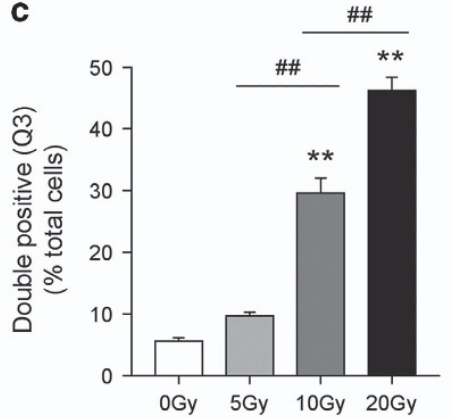

d
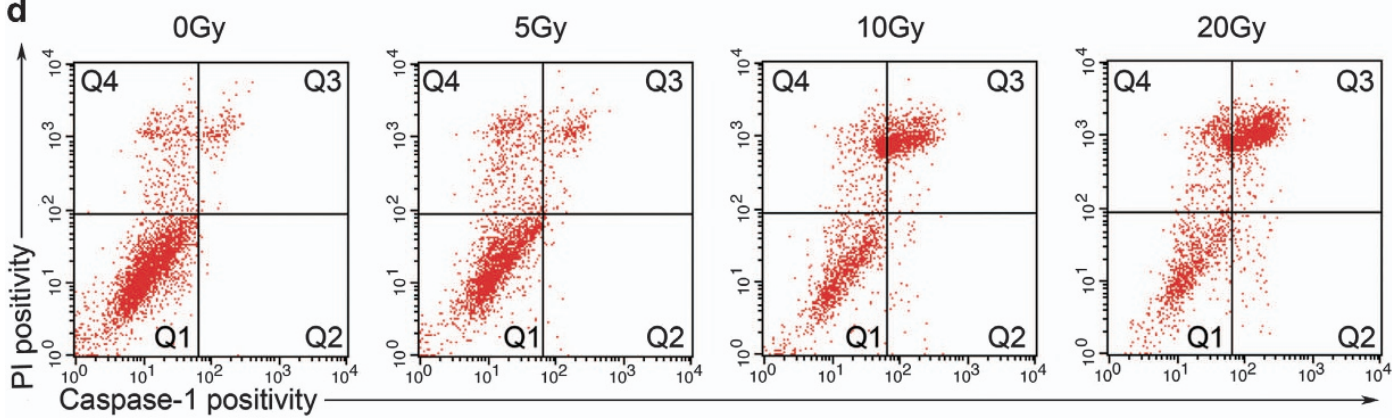

Figure 1 BMDM exposed to radiation shows an increased proportion of pyroptosis. Primarily cultured BMDM were exposed to 5, 10 and 20 Gy radiation, respectively. (a) Dose-dependent effects of radiation on the viability of BMDM. (b) LDH activity in supernatant was assessed by LDH Cytotoxicity Assay Kit, OD values of $490 \mathrm{~nm}$ were present with histogram. (c) Detected double positivity of Caspase-1 fluorescent inhibitor probe (FAM-YVAD-FMK) and PI (Q3) using flow cytometry after $24 \mathrm{~h}$. The proportions of Q3 were then presented with histogram. (d) Representative flow cytometry scatter plots. Bars represent mean \pm S.E.M. $(n=6$ or 12$)$. ${ }^{* *} P<0.001$ versus 0 Gy group; ${ }^{\#} P<0.01$, ${ }^{\# \#} P<0.001$ 

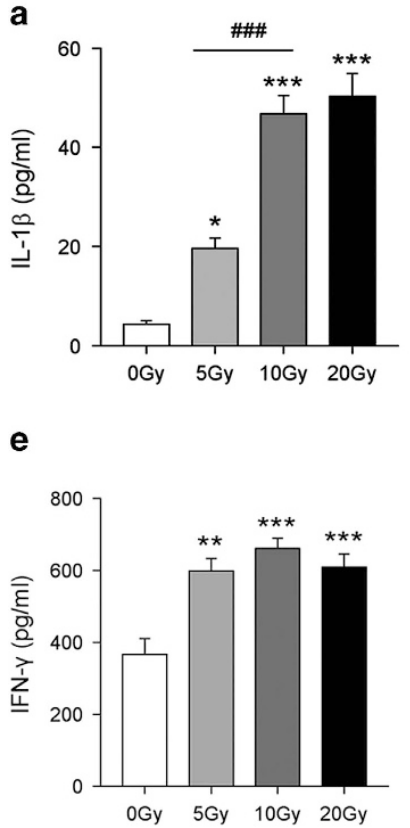

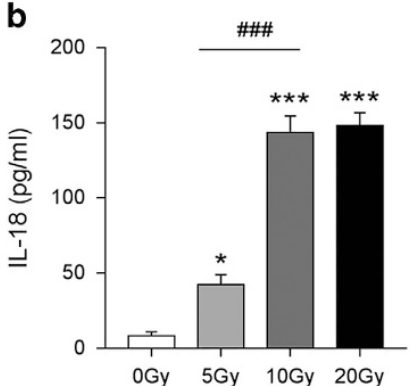

f

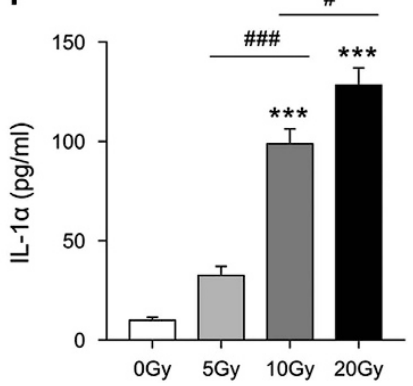

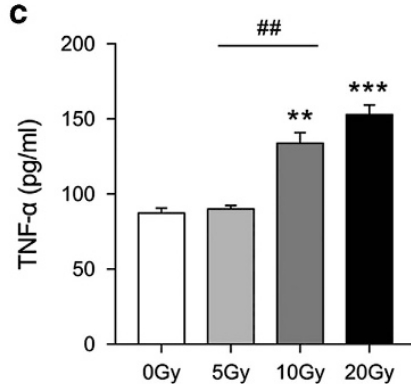

d

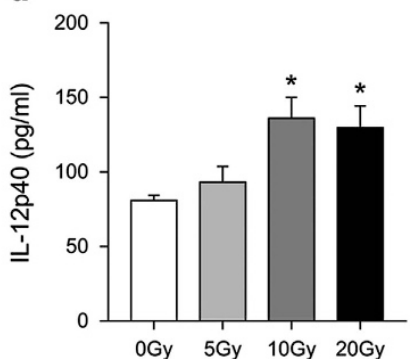

h

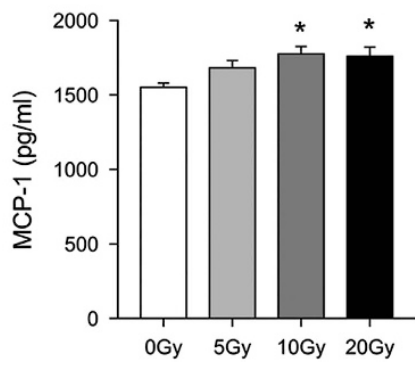

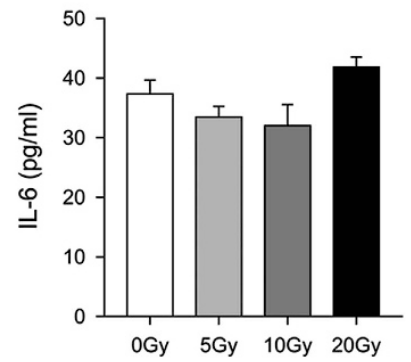

Figure 2 Radiation induces BMDM pro-inflammatory cytokine production in a dose-dependent manner. Primarily cultured BMDM were exposed to 5, 10 and 20 Gy radiation, respectively. After $24 \mathrm{~h}$, ELISA was performed to evaluate the level of pro-inflammatory cytokines in supernatant. Concentration of IL-1 $\beta$ (a), IL-18 (b), TNF- $\alpha$ (c), IL-12p40 (d),

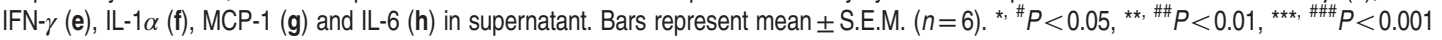

a
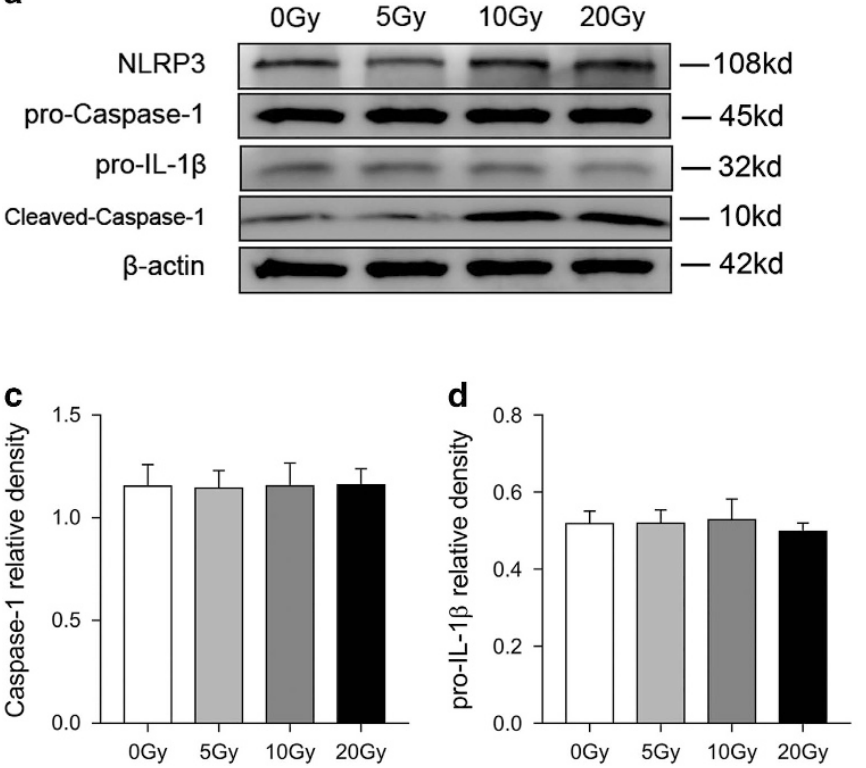
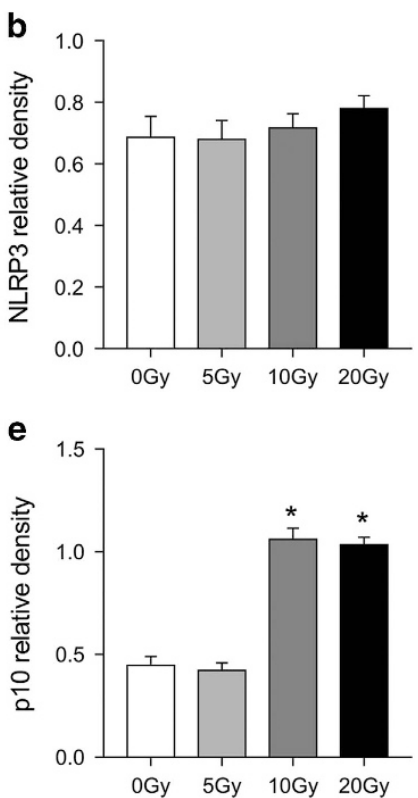

Figure 3 Radiation activates Caspase-1 in BMDM in a dose-dependent pattern. Three hours after radiation, cleared lysates of BMDM were analyzed by Western Blot. (a) Immunoblot analysis of NLRP3 inflammasome-related proteins. Relative protein levels of NLRP3 (b), Caspase-1 (c), pro-IL-1 $\beta$ (d) and cleaved-Caspase-1 (e). Band intensities were then quantified by ImageJ software and the values of the target proteins were normalized to that of $\beta$-actin. The results are representative of three independent experiments. Bars represent mean \pm S.E.M. ${ }^{*} P<0.001$

IL- $1 \beta$ and IL-18 to test the activation of Caspase- 1 in the latter study, as it was deemed more representative and economical. ${ }^{23,24}$

Radiation promoted activation of the NLRP3 inflammasome. We performed Western Blot to detect the target protein: NLRP3 (p118), precursors of Caspase-1 (pro-caspase-1, p45), precursors of IL-1 $\beta$ (pro-IL-1 $\beta$, p32) and cleaved-Caspase-1 (p10). As shown in Figure 3, 10 and 20 Gy radiation induced Caspase- 1 cleavage after $3 \mathrm{~h}$ ( $p<0.001$ versus 0 Gy) while 5 Gy radiation did not show a significant increase of cleaved-Caspase-1 ( $10, P>0.05$ versus 0 Gy). Specific comparisons (between treated groups) illustrated no significant difference in cleaved-Caspase-1 

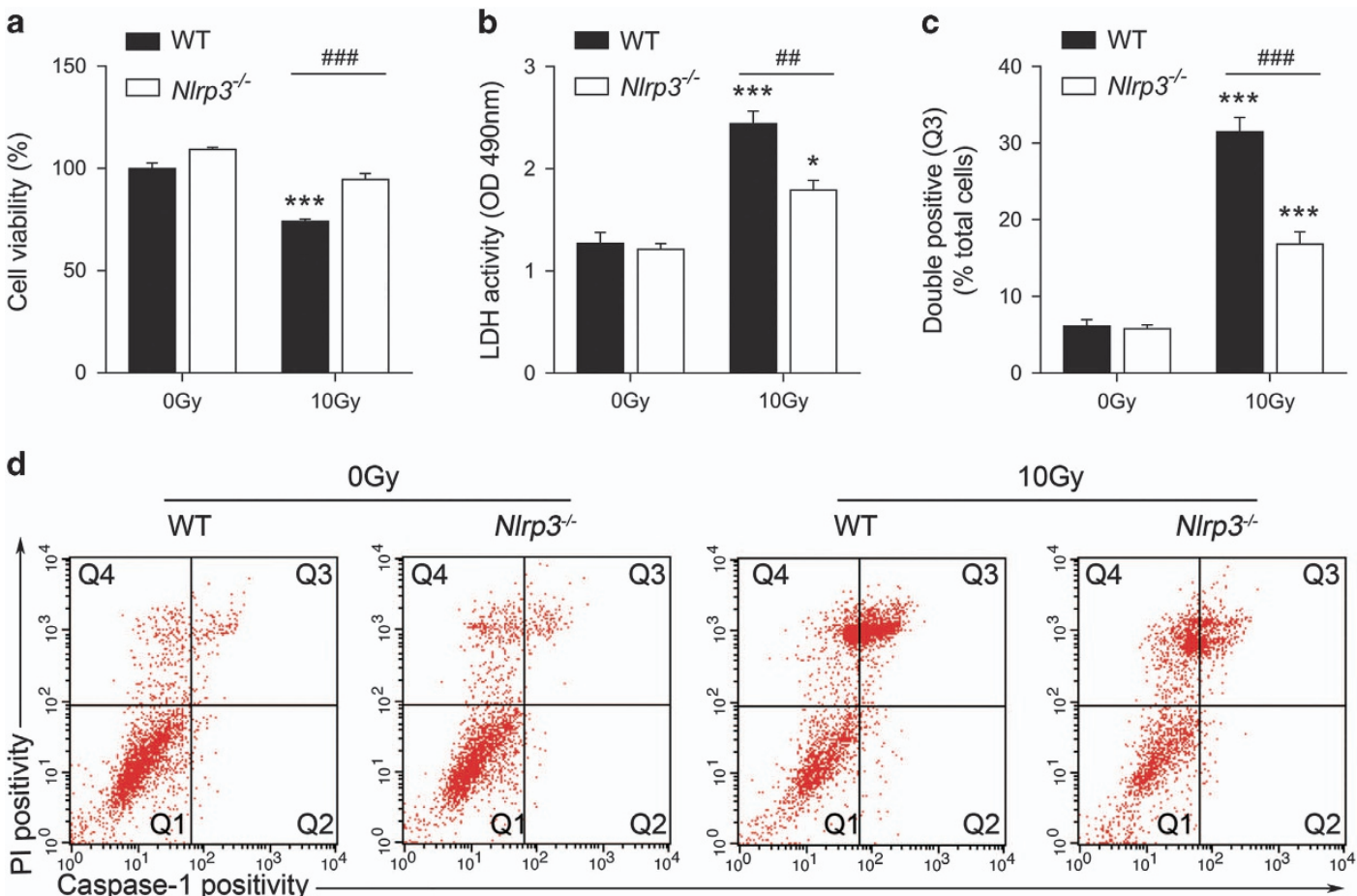

Figure 4 NIrp3 knock out inhibited radiation-induced BMDM pyroptosis. BMDM was isolated from NIrp3 ${ }^{-/-}$mice and cultured and then exposed to 10 Gy radiation. (a) Cell viability was evaluated $24 \mathrm{~h}$ after radiation. (b) Supernatant LDH activity was assessed and OD values of $490 \mathrm{~nm}$ were presented with histogram. (c) Detected double positivity of Caspase-1 fluorescent inhibitor probe (FAM-YVAD-FMK) and PI (Q3) using flow cytometry after $24 \mathrm{~h}$. The proportions of Q3 were then presented with histogram. (d) Representative flow cytometry scatter plots. Bars represent mean \pm S.E.M. $(n=6) .{ }^{*} P<0.05,{ }^{* \star *} P<0.0001$ versus 0 Gy group; ${ }^{\# \#} P<0.01,{ }^{\# \#} P<0.001$

(p10) between 10 and 20 Gy radiation groups. However, using RT-PCR, we observed that radiation did not significantly affect the mRNA levels of NLRP3 inflammasomerelated gene NIrp3, Caspase-1 or IL-1 $\beta$ ( $P>0.05$ versus 0 Gy, Supplementary Figure S1).

As the 10 Gy radiation group shows a significant effect in radiation-induced pyroptosis and Caspase- 1 activation, we chose $10 \mathrm{~Gy}$ as the treatment dosage in our subsequent research.

NIrp3 knock out suppressed radiation-induced bone marrow-derived macrophages pyroptosis and NLRP3 inflammasome activation. To determine the role of NLRP3 in radiation-induced BMDM pyroptosis, N/rp3 $3^{-1}$ BMDM was isolated from $\mathrm{NIrp3}^{-/}$mice, cultured and then exposed to10 Gy radiation. Using this strategy, we observed that NIrp3 $^{-1-}$ BMDM exhibit resistance to radiation-induced cell death (Figure 4a). Knock out of NIrp3 rescued 10 Gy induced cell death from $25.98 \%$ (wild type (WT)+10 Gy) to $5.45 \% \quad\left(N_{1 r p 3} 3^{-1}+10 \mathrm{~Gy}, \quad P<0.001 \quad \mathrm{Nlrp}^{-/}+10 \mathrm{~Gy}\right.$ versus WT+10 Gy). A test of the supernatant LDH showed that knock out of Nirp3 suppressed 10 Gy radiation induced LDH release from 2.442 (WT+10 Gy) to $1.792\left(\mathrm{Nlrp3}^{-1}+10 \mathrm{~Gy}\right.$, $P<0.01$ Nlrp3 $^{-1}+10$ Gy versus WT+10 Gy, Figure $4 \mathrm{~b}$ ). Furthermore, flow cytometry diagrams indicated that the proportion of pyroptosis induced by radiation was significantly lessened from $31.47 \%$ (WT +10 Gy) to $16.83 \%$ (NIrp3 ${ }^{-1}+10 \mathrm{~Gy}, \quad P<0.001 \quad \mathrm{Nlrp3}^{-/}+10 \mathrm{~Gy} \quad$ versus $\mathrm{WT}+10 \mathrm{~Gy}$, Figures $4 \mathrm{c}$ and d).
Immunoblotting analysis (Figures 5a-e) revealed that cleaved-Caspase-1 (p10) was elevated in WT BMDM exposed to 10 Gy radiation, while $N / r p 3^{-/}$BMDM showed no elevation of cleaved-Caspase-1 ( $p 10)$, suggesting that knock out of NIrp3 can block radiation-induced Caspase-1 activation. Likewise, knock out of NIrp3 inhibited radiation-induced IL-1 $\beta$ production $\left(P<0.001 \mathrm{Nlrp3}^{-/}+10 \mathrm{~Gy}\right.$ versus WT+10 Gy, Figure 5f).

NIrp3 knock out protected mice from radiation-induced death and Caspase-1 activation. To address whether NIrp3 knock out can overcome the lethal effect of radiation, a group of 9.5 Gy radiated $\mathrm{NIrp3}^{-/}$mice $\left(\mathrm{N} / \mathrm{rp} 3^{-/}+9.5 \mathrm{~Gy}, n=22\right)$ were monitored for 30 days in comparison with a group of 9.5 Gy radiated WT mice (WT+9.5 Gy, $n=25)$. NIrp3 ${ }^{-/}$ knockout was associated with significantly improved survival at 30 days after 9.5 Gy compared with WT+9.5 Gy group (median survival time: 27 days versus 14 days; Figure 6a, log-rank $P=0.018)$.

To further investigate the role of the NLRP3 inflammasome in radiation induced damage, cleaved-Caspase-1 (p10) and IL- $1 \beta$ were detected by Western Blot and ELISA, respectively. From Figures $6 b-g$, we observed that cleaved-Caspase-1 (p10) and IL-1 $\beta$ was induced upon radiation stimulation (both $P<0.001)$ whereas it was decreased with Nlrp3 knockout ( $P=0.0015, P<0.001$, respectively). Similar results were obtained using immunofluorescence analysis ( $3 \mathrm{~h}$ after radiation) for elevated cleaved-Caspase-1 ( $p 10$, in green fluorescence) in spleen marginal zone cells (rich in macrophages and 
a

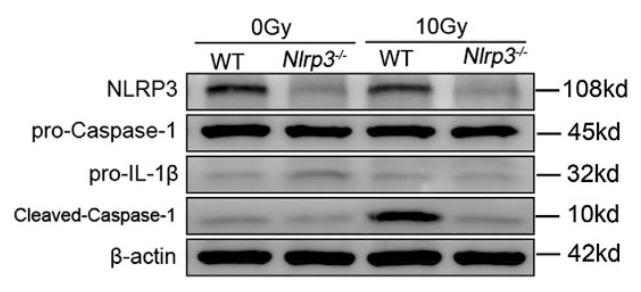

b

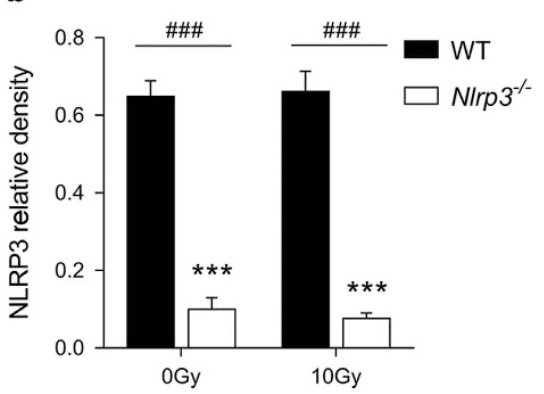

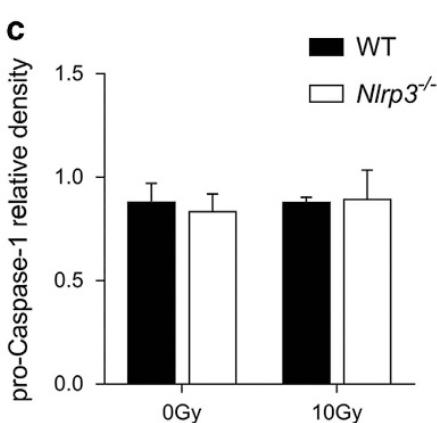
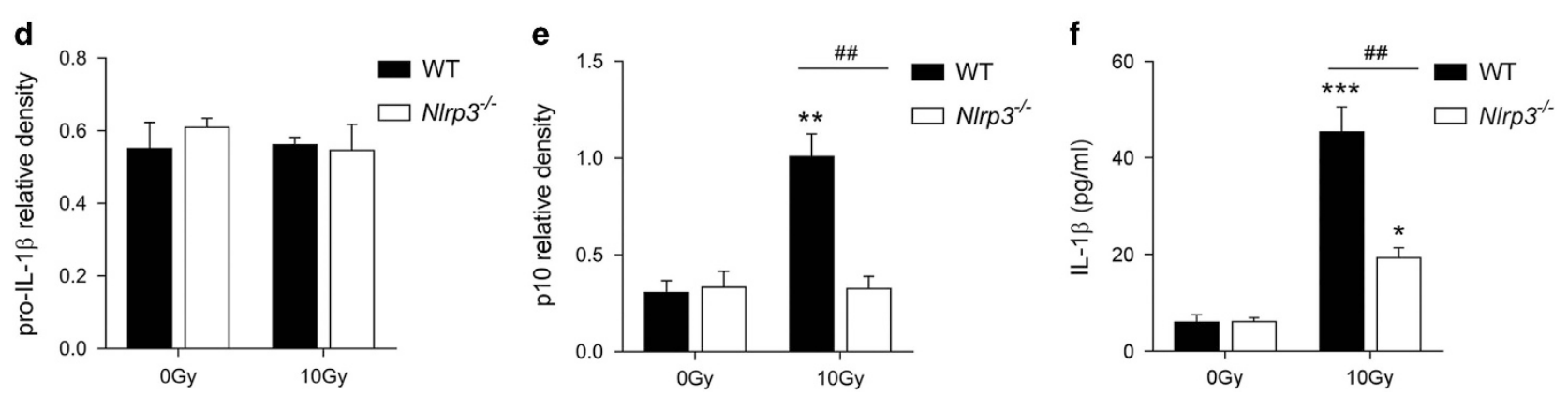

Figure 5 NIrp3 knock out inhibited radiation-induced BMDM Caspase-1 activation. (a) Western Blot analysis of NLRP3 inflammasome-related proteins. Relative protein levels of NLRP3 (b), Caspase-1 (c), pro-IL-1 $\beta$ (d) and cleaved-Caspase-1 (e). Band intensities were then quantified by ImageJ software and the values of the target proteins were normalized to that of $\beta$-actin. The results are representative of three independent experiments. (f) Concentration of IL- $1 \beta$ in supernatant. Bars represent mean \pm S.E.M. ( $n=6$ ). ${ }^{*} P<0.05,{ }^{* \star} P<0.01,{ }^{* * \star} P<0.0001$ versus 0 Gy WT group; ${ }^{\# \#} P<0.01,{ }^{\# \# \#} P<0.001$

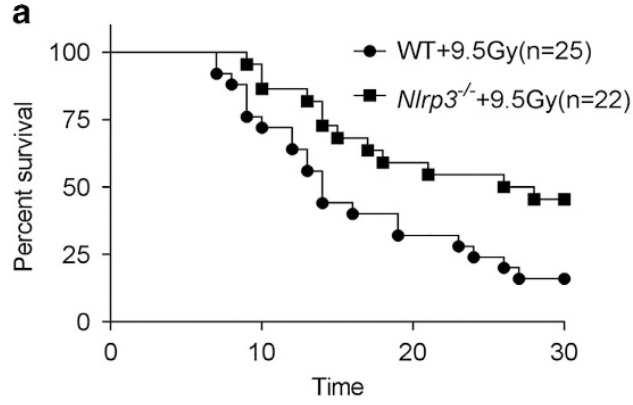

b
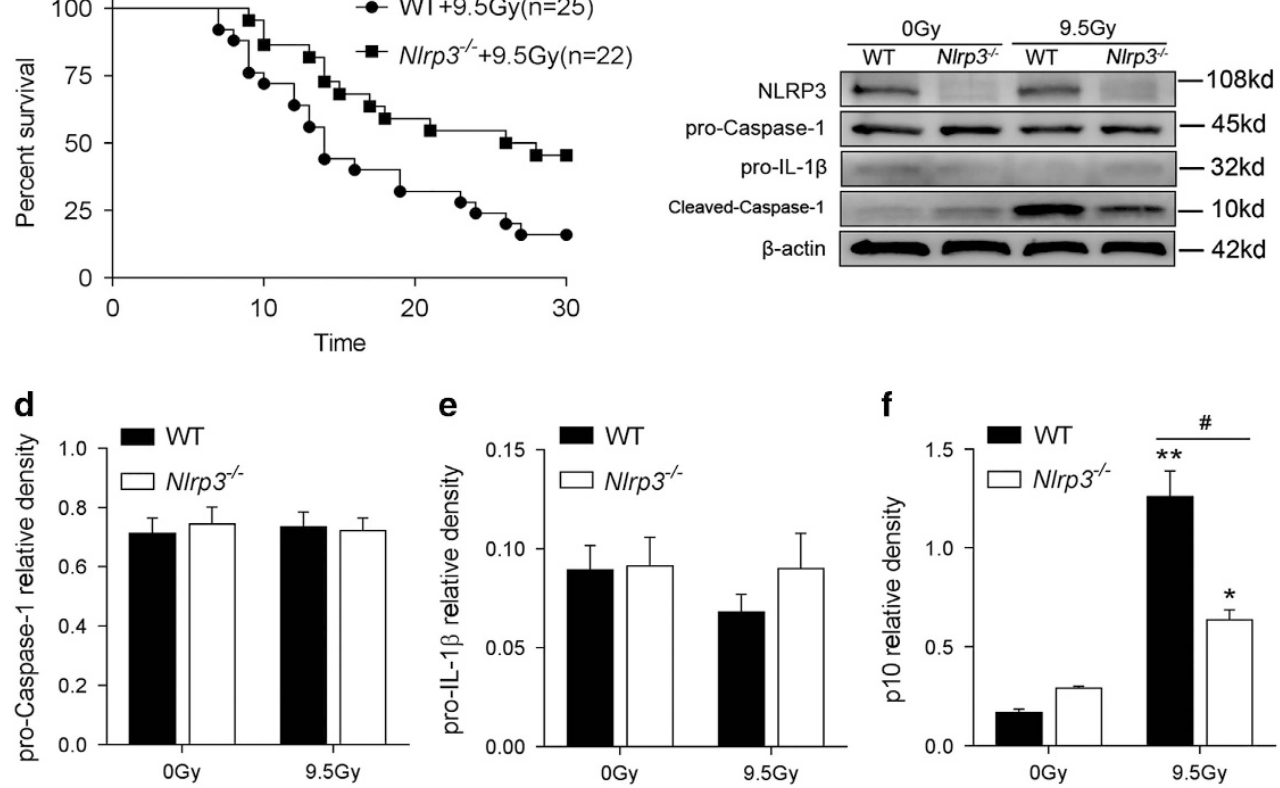
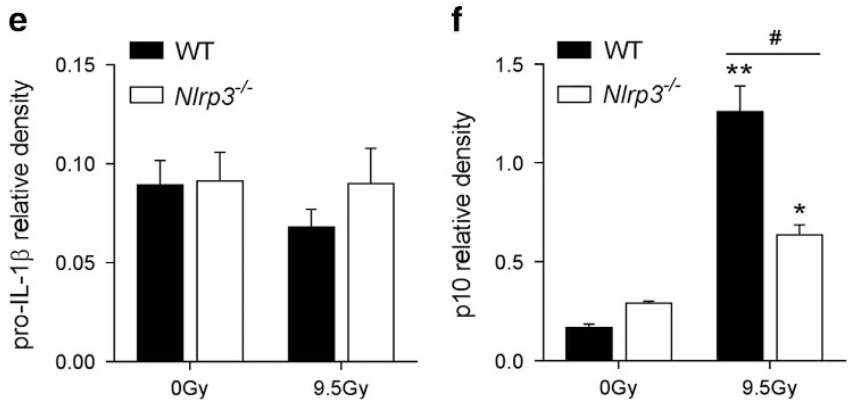

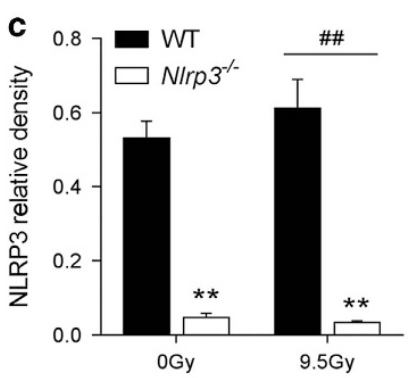

g

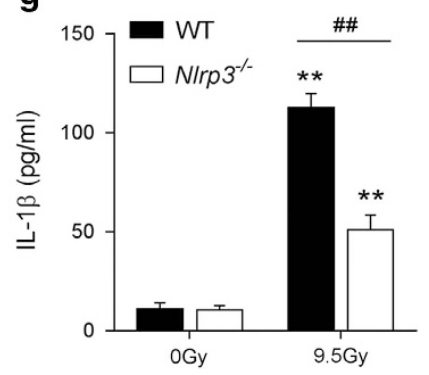

Figure 6 Nlrp3 knock out protects mice from radiation-induced death and Caspase-1 activation. (a) Survival curve: a group of 9.5 Gy radiated N/rp3 ${ }^{-/-}$mice $\left(\right.$Nlrp3 $3^{-1-}$ +9.5 Gy, $n=22$ ) were monitored for 30 days in comparison with a group of 9.5 Gy radiated WT mice (WT+9.5 Gy, $n=25)$, Log-rank (Mantel-Cox) test $P=0.0175$. (b-f) Western Blot and band intensity analysis of NLRP3-related proteins. The results are representative of three independent experiments. (g) ELISA analysis of serum IL-1 $\beta$. Bars represent mean \pm S.E.M. $(n=6-8) .{ }^{*} P<0.05,{ }^{* *} P<0.01$ versus 0 Gy WT group; ${ }^{\#} P<0.05,{ }^{\# \#} P<0.01$

follicular dendritic cells) induced by 9.5 Gy radiation. This effect can also be blocked by knocking out NIrp3 (Figures 7a and b). Notably, it was difficult to observe staining of cleaved-Caspase-1 in the white pulp cells (rich in lymphocytes) of the spleen.

\section{Discussion}

The present study demonstrates that radiation induces NLRP3 inflammasome activation and pyroptosis in BMDMs. The NLRP3 inflammasome activation, IL-1 $\beta$ production, and pyroptosis were downregulated by knockout of NIrp3. These 
a
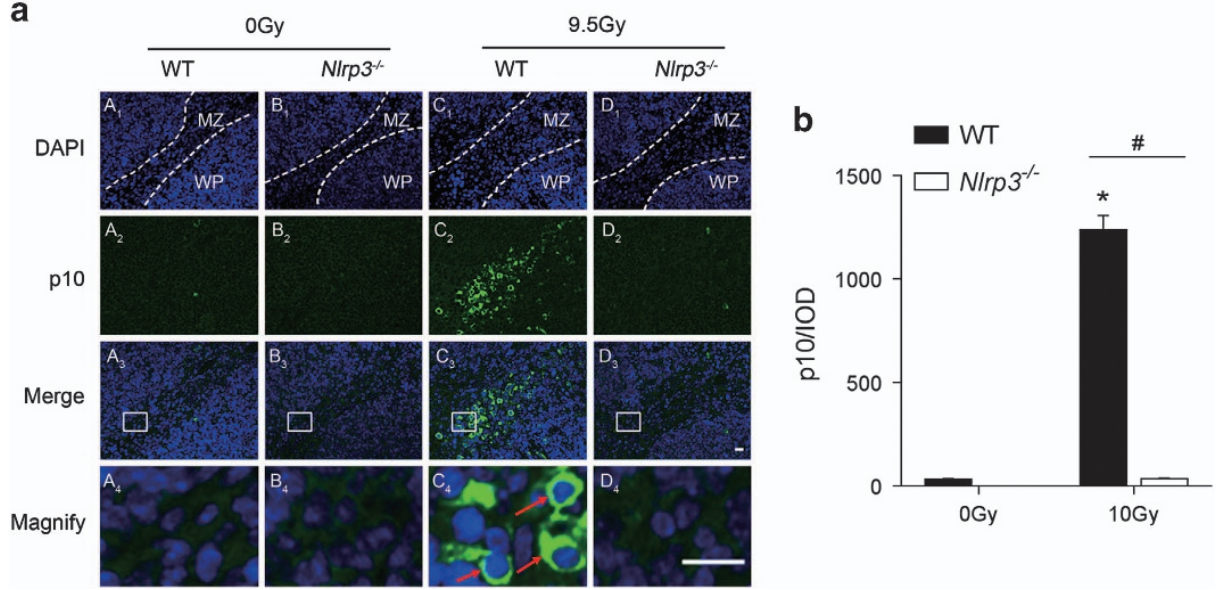

Figure 7 Immunofluorescence staining of DAPI (blue) and cleaved-Caspase-1 (p10, green) in mice spleens $3 \mathrm{~h}$ after radiation. (a) Representative microscopy images: Red arrows in magnified graph denote the fluorescence of cleaved-Caspase-1 (p10). Dotted lines indicate the border of areas; MZ, marginal zone; WP, white pulp. The results are representative of three independent experiments. Bars indicate scale of $50 \mu \mathrm{m}$. (b) Cleaved-Caspase-1 (p10) fluorescence was further quantified and presented as integrated optical density (IOD). Bars represent mean \pm S.E.M. $(n=6)$. ${ }^{*} P<0.001$ versus 0 Gy WT group; ${ }^{\#} P<0.001$

results suggest that radiation activates the NLRP3 inflammasome to induce pyroptosis and IL-1 $\beta$ production in macrophages.

Myeloid cells form the first barrier wall against inflammatory stimuli and depletion of these immune cells may damage features of the immune system and may lead to a bystander response and could result in a systemic response syndrome. ${ }^{6,25}$ However, the death modes and molecular mechanisms of immune cells exposed to radiation are only beginning to be appreciated and need further evidence to be understood. Using primary cultured BMDMs, our study demonstrated that radiation induces macrophage death and pro-inflammatory cytokine (includes M1 cytokines such as TNF- $a$, IFN- $\gamma$ and IL-12) release in a dose-dependent manner (Figures 1 and 2), which is broadly consistent with previous studies. ${ }^{5,9,10}$ We found a significant increase in MCP-1 in radiation treated groups, which was also shown by DeBo et al., although not in Stoecklein's or Siva's research. ${ }^{26,27}$ Additionally, we did not detect obvious increases in IL- 6 after radiation, which is not consistent with several previous studies. ${ }^{28}$ These differences in findings may be because of the use of different cell types and/or radiation sources.

Pyroptosis, defined as Caspase-1-dependent programmed and pro-inflammatory cell death, is distinct from any other programmed cell death and results in cell lysis and proinflammatory cytokine release. ${ }^{29}$ Pyroptosis is triggered by various pathological stimuli, such as stroke, heart attack, cancer, microbial or liver inflammation and fibrosis. ${ }^{30,31}$ Unlike Caspase-3-dependent apoptosis, pyroptosis is typically mediated by Caspase- 1 activation following inflammasome complex formation. ${ }^{16}$ Another apparent feature of pyroptosis is pore formation, which permits cytosolic contents such as LDH and pro-inflammatory cytokine release and fluorochromeconjugated annexin V, 7-aminoactinomycin (7-AAD) or $\mathrm{PI}$ enter the cell. ${ }^{13}$ Nevertheless, membrane impermeant dyes such as 7-AAD or PI do not stain apoptotic cells. ${ }^{13}$ Although Stoecklein et al. discussed the role of radiation-induced immune cell pyroptosis in their good work, there remains some confusion about the methods of pyroptosis detection. ${ }^{5}$ Stoecklein et al. first stained cells with annexin $V$ and cleavedCaspase-1 ( 10 ) and found annexin $\mathrm{V}^{+}$cells express higher cleaved-Caspase-1 ( $p 10$ ) than annexin $\mathrm{V}^{-}$cells after radiation. Then, they stained another group of radiated cells with annexin $\mathrm{V}$ and $\mathrm{PI}$ and found that radiation increased doublepositive cells (annexin $\mathrm{V}^{+} / \mathrm{PI}^{+}$). Taken together, they concluded that these annexin $\mathrm{V}^{+} / \mathrm{PI}^{+}$cells may also express more cleaved-Caspase-1 (p10) and be more subject to pyroptotic death. However, in an indirect way, the method cannot exclude

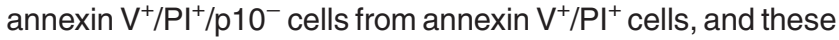
type of cells (annexin $\mathrm{V}^{+} / \mathrm{PI}^{+} / \mathrm{p} 10^{-}$) are known as apoptotic cells in the late stage, not pyroptotic cells. Indeed, although controversial, many studies have used relatively direct and exact methods to detect pyroptosis such as double staining cells with FAM-YVAD-FMK (a marker of activated Caspase-1) and PI or SYTOX Blue DNA intercalation staining (a marker of pore formation in the plasma membrane). ${ }^{30,32}$ Detection of $\mathrm{LDH}$ release (as a marker of pore formation in the plasma membrane) is also used widely to identify pyroptosis. ${ }^{32}$ Some labs like Jiahuai et al.'s and Lei et al.'s also have made great efforts to explore the morphologic characteristics of pyroptosis by electron microscopy and have made certain achievements recently that may contribute to the future detection of pyroptosis. $^{30,33}$ In this study, detection of active caspase 1 and PI double stain were used in combination with an LDH release assay to detect and quantify pyroptosis. Our current data confirmed that radiation induces BMDM pyroptosis in a dose-dependent manner. Importantly, the 5 Gy radiation group did not show a significant increase of double-positive Caspase-1 and PI, nor did they show an elevation of LDH activity compared with the control group (0 Gy), which suggests a BMDM tolerance to relatively low doses of radiation (Figure 1). Many studies have shown an active role for macrophages under relatively low doses of radiation (2 Gy or $8 \mathrm{~Gy}$ ) when many other cell types exhibit a marked loss. ${ }^{34-36}$ Based on these results and previous research, we favor the hypothesis that low dose radiation activates the 
macrophages that then mediate a variety of biological effects while relative high dose radiation kills the macrophages in a pyroptotic manner and mediates various types of severe damage.

As pyroptosis occurs after activation of caspase-1 and many studies that have focused on the inflammatory responses under radiation detected controversial results about the links between radiation exposure and NLRP3 inflammasome activation, we then tested the role of the NLRP3 inflammasome in radiation and in the pyroptosis induced by radiation in cultured BMDM using RT-PCR, immunoblotting and ELISA techniques. Interestingly, the results imply that BMDM exposed to radiation shows evidence of dose-dependent Caspase- 1 activation and IL-1 $\beta$ production (Figures 2 and 3 ) and that the Caspase- 1 activation and $\mathrm{IL}-1 \beta$ production is dependent on NLRP3, since NIrp3 knock out diminishes the quantity of cleaved-Caspase-1 (p10) and IL-1 $\beta$ (Figures 4 and 5). This conclusion was confirmed by in vivo research (Figures 6 and 7). More vitally, Nlrp3 knock out can also significantly lessen the proportion of pyroptosis induced by radiation. Accordingly, we believe that radiation can induce pyroptosis through activating the NLRP3 inflammasome. This finding is novel and further supports the idea that NLRP3Caspase-1 inflammasome activation is essential in radiation induced cell and tissue damage. ${ }^{19,21}$ Whereas, we noticed that depletion of NLRP3 rescued only a fraction of BMDM from radiation induced death and pyroptosis and Caspase-1 activation, which supports the hypothesis that radiationinduced Caspase-1 activation and pyroptosis is not wholly NLRP3-dependent. As radiation can break gut mucosa and lung epithelial cells which, in turn, increase susceptibility to infections, other inflammasomes such as NLRP1, NLRC4 or AIM2 that can recognize PAMPs may also contribute to radiation-induced caspase-1 activation. ${ }^{21,37,38}$ Stoecklein found that $\mathrm{NIrp3}^{-/}$mice did not show a protective effect against radiation-induced immune cell loss. This could be partially explained by the fact that relative low dose radiation induces immune cell loss indirectly, such as through PAMPs activating other inflammasomes, which further supports our previous inference. It is also worth noting that, we did not observe a significant change in the expression of NLRP3 inflammasome-related protein NIrp3, Caspase-1 and IL-1 $\beta 3 \mathrm{~h}$ after radiation (Supplementary Figure S1; Figure 3 ), while other studies had detected elevated expression levels of the NLRP3 inflammasome-related protein after at least 1 day. ${ }^{18-20}$ The activation of the NLRP3 inflammasome is generally believed to require two signals, signal 1 being Toll-like receptor activation leading to cellular priming and upregulation of NLRP3 expression and signal 2 being an additional stimulation of these cells with PAMPs or DAMPs. ${ }^{39}$ As we know, protein expression is time-dependent and those studies that detected elevated NLRP3 inflammasome related protein at least $24 \mathrm{~h}$ after radiation cannot avoid the effect of radiationinduced cytokines on NLRP3 inflammasome-related protein expression. Indeed, BMDM constitutively express NLRP3 which make it possible for agonists to directly activate NLRP3 inflammasome. ${ }^{40}$ The direct effects of radiation, such as damage to lysosomes, nuclear DNA or mitochondrial DNA, could be the signal 2 of NLRP3 inflammasome activation. ${ }^{41}$ In addition, different tissue or cell types may react differently to iron radiation. ${ }^{42}$ Based on these findings, we assume that radiation does not directly affect the expression of NLRP3 inflammasome-related protein in the early stage.

To the best of our knowledge, we reported here for the first time that NLRP3 inflammasome activation mediates radiationinduced pyroptosis in BMDMs. We further assume that radiation can directly activate the NLRP3 inflammasome without affecting the expression level and indirectly activate other inflammasomes in the later period. Targeting the NLRP3 inflammasome and secondary pyroptosis may represent a novel strategy to limit the radiation-induced loss of immune cells, cascades of pro-inflammatory cytokines and related tissue damage. However, there are still limitations that need further exploration. For example, the mechanism of radiation activation of the NLRP3 inflammasome, the downstream cytokine induction of Caspase-1 activation and the role of other inflammasomes in radiation induced cell or tissue damage are not clear.

\section{Materials and Methods}

Animals. WT C57BL/6J male mice, 5-7 weeks age, were purchased from SuperB\&K Laboratory Animal Corp. Ltd. (Shanghai, China). Male NIrp3 ${ }^{-1-}$ mice on the C57BL/6J genetic background were from Model Animal Research Centre (AAALAC accredited, Nanjing University, China). Housed in specific-pathogen-free animal facility for at least 5 days under a 12-h light/dark cycle, the animals were given access to water and standard laboratory chow ad libitum. Those knockout mice and cells were identified by PCR (sequences of primers were presented in Supplementary Table S2), DNA sequencing, immunoblotting and immunofluorescence staining (Supplementary Figure S2). All animal procedures used in this study were approved by the Institutional Animal Care and Use Committee of the Second Military Medical University (No. 20120025, Shanghai, China).

Cells isolation and culture. The isolation and culture of BMDM was performed as Pineda-Torra et al. described. ${ }^{43}$ At first, briefly, animals were killed by cervical dislocation and soaked in $75 \%$ ethanol. Then, femurs and tibias were harvested and the bone marrow cells from all bones were flushed out. After centrifuging for 5 min at $310 \times g$, Erythrocytes were eliminated using Red Blood Cell Lysing Buffer (Sigma-Aldrich, St. Louis, MO, USAhttp://www.sigmaaldrich.com).The remaining cells were seeded in plates and incubated in complete medium with $50 \mathrm{mg} / \mathrm{ml}$ recombinant mouse M-CSF (R\&D Systems, Inc., Minneapolis, MN, USA) for 7 days to form proliferative nonactivated cells (also named MO macrophages).

$\boldsymbol{\gamma}$-ray radiation. In vivo, mice were exposed to whole-body radiation by timed exposure to ${ }^{60} \mathrm{Co}$ radiation source (Faculty of Naval Medicine, Second Military Medical University) with a dose rate of $1.63 \mathrm{~Gy} / \mathrm{min}$ and cumulative radiation dosage of 9.50 Gy. For in vitro assay, BMDM were grown as a monolayer and were exposed to ${ }^{60} \mathrm{Co}$ radiation source to attain the desired dose of 5, 10 and $20 \mathrm{~Gy}$ at a dose rate of $1.80 \mathrm{~Gy} / \mathrm{min}$.

Cell viability assay. Thirty thousand cells per well were seeded into 96-well plates in $100 \mu \mathrm{l}$ of RPMl 1640 supplemented with $10 \%$ FBS, $0.1 \mathrm{mg} / \mathrm{ml}$ Penicillin/ Streptomycin (P/S) and $50 \mathrm{mg} / \mathrm{ml}$ recombinant mouse M-CSF and incubated for 7 days as described before. Then, the cells were exposed to ${ }^{60} \mathrm{Co}$ radiation and the number of surviving cells was measured by Cell Counting Kit-8 (CCK-8) (Dojindo Laboratories, Kumamoto, Japan) after $24 \mathrm{~h}$. Data acquisition was performed on DENLEY DRAGON Wellscan MK 3 (Thermo, Vantaa, Finland). Cell viability was calculated according to the formula: cell viability $(\%)=[(A s-A b) /(A c-A b) \times 100 \%$, where $A s, A c$ and $A b$ represent the $A 450$ in treated, untreated and blank groups, respectively.

Determination of BMDM pyroptosis. Pyroptotic cell death was assessed by measuring the release of $\mathrm{LDH}$ and double-positive staining of activated Caspase1 and PI in BMDM. Released LDH in the cell culture supernatant was detected using LDH Cytotoxicity Assay Kit (Beyotime, Haimen, Jiangsu, China) and the activated Caspase-1 and PI were detected by FAM-FLICA Caspase-1 Assay Kit (ImmunoChemistry Technologies, LCC, Bloomington, MN, USA) according to the 
manufacturer's instruction. Stained cells were then analyzed by flow cytometry (BD FACSCalibur, Becton, Dickinson and Company, Franklin Lakes, NJ, USA)

Quantitative real-time polymerase chain reaction (qRT-PCR). The total RNA was isolated from adhered BMDM ( $3 \mathrm{~h}$ after radiation) using Trizol reagent (Life Technologies, Carlsbad, CA, USA). The reverse transcript (cDNA) was synthesized from $1 \mu \mathrm{g}$ of total RNA and PrimeScript RT Master Mix (Takara Biotechnology, Dalian, China). QRT-PCR was performed with $2 \mu$ l first-strand cDNA solution in combination with a Fast start Universal Probe Master Mix, in a final volume of $20 \mu \mathrm{l}$. The primers used in this study are listed in Supplementary Table S1. All samples were run in triplicate and underwent 40 amplification cycles on an Applied Biosystems 7500 Real-Time PCR System (Life Technologies Corporation, USA) following the manufacturer's protocols. Expression values of NLRP3, Caspase1 and pro-IL-1 $\beta$ were normalized to the value of the endogenous standard GAPDH and calculated by using the comparative cycle threshold $\left(\Delta \Delta \mathrm{C}_{t}\right)$ method. ${ }^{44}$

Immunoblot analysis. For Western Blot analysis, adhered BMDM $(3 \mathrm{~h}$ after radiation) or spleens of mice ( $3 \mathrm{~h}$ after radiation) were lysed and the protein concentrations were measured as we described previously. ${ }^{45}$ Cleared lysates were separated by $10 \%$ SDS-PAGE, transferred onto NC membranes and then blocked for $2 \mathrm{~h}$ at room temperature with $5 \%$ nonfat dried milk. NLRP3, pro-Caspase- 1 (p45) and cleaved-Caspase-1 ( $p 10)$ detection was accomplished by probing the membranes with anti-NLRP3 Ab (AdipoGen Corp., San Diego, CA, USA), antiCaspase-1 (p45) Ab (Abcam, Cambridge, UK) and anti-cleaved-Caspase-1 (p10) Ab (Santa Cruz Biotechnology, Inc., Dallas, TX, USA), and exposed with an Amersham Imager 600 (GE Healthcare Bio-Sciences AB, Uppsala, Sweden). The membranes were then stripped, reprobed with anti- $\beta$-actin and exposed again to detect the endogenous standard $\beta$-actin. ImageJ software was then used to scan and quantify the immunoblots. The band intensity values of the target proteins were normalized to that of $\beta$-actin.

Cytokine measurement. Cell-free culture supernatants were tested for IL-1 $\beta$ IL-18 IL-1 $\alpha$, TNF- $\alpha$, IFN- $\gamma$, IL-6, IL-12p40 and MCP-1 concentrations using ELISA kits (Dakewe Biotech Company Ltd., Shenzhen, China) following the manufacturer's protocols. Data acquisition was performed on DENLEY DRAGON Wellscan MK 3 (Thermo, Finland).

Immunofluorescence staining. After anesthetizing, the mice were transcardially perfused with normal saline $(0.9 \%)$ and spleen tissues were isolated and fixed in fresh paraformaldehyde solution (4\%, PH 7.4). Horizontal slices were prepared and blocked with $1 \%$ bovine serum albumin (BSA; Sigma-Aldrich) in PBS containing $0.05 \%$ Tween $20(0.05 \%$ PBS-T) for $2 \mathrm{~h}$. Specimens were then incubated with primary described anti-cleaved-Caspase- 1 (p10) Ab overnight at $4{ }^{\circ} \mathrm{C}$ and Alexa Fluor 488 (green) labeled donkey anti-rabbit lgG were then loaded for $2 \mathrm{~h}$ at room temperature in the dark. After washing, DAPI (4',6-diamidino-2-phenylindole) was used for nuclear staining. Stained specimens were visualized with an Olympus Research Inverted System Microscope IX71 (Olympus, Tokyo, Japan). Fluorescence intensity of cleaved-Caspase-1 (p10) was quantified by Image Pro Plus 6.0 software (Media Cybernetics, Silver Spring, MD, USA).

Statistical analysis. The data are expressed as the mean \pm S.E.M. and analyzed for statistical significance using GraphPad Prism 5.0.1 (GraphPad Software, La Jolla, CA, USA). One-way ANOVA was used to detected statistical significance among group means and Bonferroni post-hoc analysis was used to compare specific groups when ANOVA showed significant differences. $P<0.05$ was considered to be statistically significant.

\section{Conflict of Interest}

The authors declare no conflict of interests.

Acknowledgements. This work was supported by the Major Project of National Science and Technology (2014ZX09J14106-06C, 13CXZ005), the National Natural Science Foundation of China $(81473291,81402651,81402654)$ and the Natural Science Foundation of Shanghai (13ZR144940, 16ZR1444500).

1. Wilkins A, Mossop H, Syndikus I, Khoo V, Bloomfield D, Parker C et al. Hypofractionated radiotherapy versus conventionally fractionated radiotherapy for patients with intermediate- risk localised prostate cancer: 2-year patient-reported outcomes of the randomised, non-inferiority, phase $3 \mathrm{CHHiP}$ trial. Lancet Oncol 2015; 16: 1605-1616.

2. Bradley JD, Paulus R, Komaki R, Masters G, Blumenschein G, Schild S et al. Standard-dose versus high-dose conformal radiotherapy with concurrent and consolidation carboplatin plus paclitaxel with or without cetuximab for patients with stage IIIA or IIIB non-small-cell lung cancer (RTOG 0617): a randomised, two-by-two factorial phase 3 study. Lancet Oncol 2015; 16: $187-199$

3. De Meerleer G, Khoo V, Escudier B, Joniau S, Bossi A, Ost P et al. Radiotherapy for renalcell carcinoma. Lancet Oncol 2014; 15: e170-e177.

4. Kim JH, Jenrow KA, Brown SL. Mechanisms of radiation-induced normal tissue toxicity and implications for future clinical trials. Radiat oncol $j 2014 ; 32$ : 103-115.

5. Stoecklein VM, Osuka A, Ishikawa S, Lederer MR, Wanke-Jellinek L, Lederer JA. Radiation exposure induces inflammasome pathway activation in immune cells. J immunol 2015; 194 : 1178-1189.

6. Ratikan JA, Micewicz ED, Xie MW, Schaue D. Radiation takes its toll. Cancer lett 2015; 368 : 238-245.

7. Ahn GO, Tseng D, Liao CH, Dorie MJ, Czechowicz A, Brown JM. Inhibition of Mac-1 (CD11b/CD18) enhances tumor response to radiation by reducing myeloid cell recruitment. Proc Natl Acad Sci USA 2010; 107: 8363-8368.

8. Cappuccini F, Eldh T, Bruder D, Gereke M, Jastrow H, Schulze-Osthoff $\mathrm{K}$ et al. New insights into the molecular pathology of radiation-induced pneumopathy. Radiother oncol 2011; 101: 86-92.

9. Farooque A, Afrin F, Adhikari JS, Dwarakanath BS. Polarization of macrophages towards M1 phenotype by a combination of 2-deoxy-d-glucose and radiation: implications for tumor therapy. Immunobiology 2016; 221: 269-281.

10. Klug F, Prakash H, Huber PE, Seibel T, Bender N, Halama N et al. Low-dose irradiation programs macrophage differentiation to an $\mathrm{iNOS}(+) / \mathrm{M} 1$ phenotype that orchestrates effective T cell immunotherapy. Cancer cell 2013; 24: 589-602.

11. Malhotra P, Adhikari M, Mishra S, Singh S, Kumar P, Singh SK et al. N-acetyl tryptophan glucopyranoside (NATG) as a countermeasure against gamma radiation-induced immunosuppression in murine macrophage J774A.1 cells. Free rad res 2016; 50: 1-14.

12. Abe J, Morrell C. Pyroptosis as a regulated form of necrosis: $\mathrm{PI}+$ /annexin $\mathrm{V}-$ /high caspase 1/low caspase 9 activity in cells = pyroptosis? Circ res 2016; 118: 1457-1460.

13. Miao EA, Rajan JV, Aderem A. Caspase-1-induced pyroptotic cell death. Immunol rev 2011; 243: 206-214.

14. Croker BA, O'Donnell JA, Gerlic M. Pyroptotic death storms and cytopenia. Curr opin immunol 2014; 26: 128-137.

15. Guo H, Callaway JB, Ting JP. Inflammasomes: mechanism of action, role in disease, and therapeutics. Nature med 2015; 21: 677-687.

16. Aachoui Y, Sagulenko V, Miao EA, Stacey KJ. Inflammasome-mediated pyroptotic and apoptotic cell death, and defense against infection. Curr opin microbiol 2013; 16: 319-326.

17. Brennan MA, Cookson BT. Salmonella induces macrophage death by caspase-1-dependent necrosis. Mol microbiol 2000; 38: 31-40.

18. Sohn SH, Lee JM, Park S, Yoo H, Kang JW, Shin D et al. The inflammasome accelerates radiation-induced lung inflammation and fibrosis in mice. Environ toxicol pharmacol 2015; 39 : 917-926.

19. Ortiz F, Acuna-Castroviejo D, Doerrier C, Dayoub JC, Lopez LC, Venegas C et al. Melatonin blunts the mitochondrial/NLRP3 connection and protects against radiation-induced oral mucositis. J pineal res 2015; 58: 34-49.

20. Shin D, Lee G, Sohn SH, Park S, Jung KH, Lee JM et al. Regulatory T cells contribute to the inhibition of radiation-induced acute lung inflammation via bee venom phospholipase $A(2)$ in mice. Toxins 2016; 8: 131.

21. Feldmeyer L, Keller M, Niklaus G, Hohl D, Werner S, Beer HD. The inflammasome mediates UVB-induced activation and secretion of interleukin-1beta by keratinocytes. Curr biol 2007; 17: $1140-1145$

22. Li T, Li L, Li F, Liu Y. X-ray irradiation accelerates senescence in hippocampal neural stem/ progenitor cells via caspase-1 activation. Neurosci lett 2015; 585: 60-65.

23. Sheedy FJ, Grebe A, Rayner KJ, Kalantari P, Ramkhelawon B, Carpenter SB et al. CD36 coordinates NLRP3 inflammasome activation by facilitating intracellular nucleation of soluble ligands into particulate ligands in sterile inflammation. Nature immunol 2013; 14 : 812-820.

24. Huai W, Zhao R, Song H, Zhao J, Zhang L, Zhang L et al. Aryl hydrocarbon receptor negatively regulates NLRP3 inflammasome activity by inhibiting NLRP3 transcription. Nature commun 2014; 5: 4738.

25. Prise KM, Schettino G, Folkard M, Held KD. New insights on cell death from radiation exposure. Lancet Oncol 2005; 6: 520-528.

26. DeBo RJ, Lees CJ, Dugan GO, Caudell DL, Michalson KT, Hanbury DB et al. Late effects of total-body gamma irradiation on cardiac structure and function in male rhesus macaques. Radiat res 2016; 186: 55-64.

27. Siva S, MacManus M, Kron T, Best N, Smith J, Lobachevsky $P$ et al. A pattern of early radiation-induced inflammatory cytokine expression is associated with lung toxicity in patients with non-small cell lung cancer. PloS one 2014; 9: e109560.

28. Oh ET, Park MT, Song MJ, Lee H, Cho YU, Kim SJ et al. Radiation-induced angiogenic signaling pathway in endothelial cells obtained from normal and cancer tissue of human breast. Oncogene 2014; 33: 1229-1238.

29. LaRock CN, Cookson BT. Burning down the house: cellular actions during pyroptosis. PLoS pathog 2013; 9: e1003793. 
30. Geng Y, Ma Q, Liu YN, Peng N, Yuan FF, Li XG et al. Heatstroke induces liver injury via IL-1beta and HMGB1-induced pyroptosis. J hepatol 2015; 63: 622-633.

31. Wree A, Eguchi A, McGeough MD, Pena CA, Johnson CD, Canbay A et al. NLRP3 inflammasome activation results in hepatocyte pyroptosis, liver inflammation, and fibrosis in mice. Hepatology (Baltimore, Md) 2014; 59: 898-910.

32. Chen H, Lu Y, Cao Z, Ma Q, Pi H, Fang Y et al. Cadmium induces NLRP3 inflammasomedependent pyroptosis in vascular endothelial cells. Toxicol lett 2016; 246: 7-16.

33. Chen X, He WT, Hu L, Li J, Fang Y, Wang X et al. Pyroptosis is driven by non-selective gasdermin-D pore and its morphology is different from MLKL channel-mediated necroptosis. Cell res 2016; 26: 1007-1020.

34. De Palma M, Coukos G, Hanahan D. A new twist on radiation oncology: low-dose irradiation elicits immunostimulatory macrophages that unlock barriers to tumor immunotherapy. Cancer cell 2013; 24: 559-561.

35. Seifert L, Werba G, Tiwari S, Giao Ly NN, Nguy S, Alothman S et al. Radiation therapy induces macrophages to suppress T-cell responses against pancreatic tumors in mice. Gastroenterology 2016; 150: 1659-1672.e1655.

36. Mukherjee D, Coates PJ, Lorimore SA, Wright EG. Responses to ionizing radiation mediated by inflammatory mechanisms. J pathol 2014; 232: 289-299.

37. Coggle JE, Lambert BE, Moores SR. Radiation effects in the lung. Environ health perspect 1986; 70: 261-291.

38. Francois A, Milliat F, Guipaud $\mathrm{O}$, Benderitter M. Inflammation and immunity in radiation damage to the gut mucosa. BioMed res int 2013; 2013: 123241.

39. Anand PK, Malireddi RK, Kanneganti TD. Role of the nlrp3 inflammasome in microbial infection. Front microbiol 2011; 2: 12.

40. Chen M, Wang $\mathrm{H}$, Chen W, Meng G. Regulation of adaptive immunity by the NLRP3 inflammasome. Int immunopharmacol 2011; 11: 549-554.

41. Sutterwala FS, Haasken S, Cassel SL. Mechanism of NLRP3 inflammasome activation. Ann NY Acad Sci 2014; 1319: 82-95.
42. Vianale G, Reale M, Amerio P, Stefanachi M, Di Luzio S, Muraro R. Extremely low frequency electromagnetic field enhances human keratinocyte cell growth and decreases proinflammatory chemokine production. Br j dermatol 2008; 158: 1189-1196.

43. Pineda-Torra I, Gage M, de Juan A, Pello OM. Isolation, culture, and polarization of murine bone marrow-derived and peritoneal macrophages. Meth mol biol 2015; 1339: 101-109.

44. Lebeaupin C, Proics E, de Bieville $\mathrm{CH}$, Rousseau D, Bonnafous S, Patouraux S et al. ER stress induces NLRP3 inflammasome activation and hepatocyte death. Cell death dis 2015; 6: e1879.

45. Zhang ZT, Du XM, Ma XJ, Zong Y, Chen JK, Yu CL et al. Activation of the NLRP3 inflammasome in lipopolysaccharide-induced mouse fatigue and its relevance to chronic fatigue syndrome. J neuroinflammation 2016; 13: 71.

Cell Death and Disease is an open-access journal published by Nature Publishing Group. This work is

licensed under a Creative Commons Attribution 4.0 International License. The images or other third party material in this article are included in the article's Creative Commons license, unless indicated otherwise in the credit line; if the material is not included under the Creative Commons license, users will need to obtain permission from the license holder to reproduce the material. To view a copy of this license, visit http://creativecommons.org/licenses/by/4.0/

(C) The Author(s) 2017

Supplementary Information accompanies this paper on Cell Death and Disease website (http://www.nature.com/cddis) 